04

\title{
Величина и знак электростатического заряда, потенциала и напряженности магнитного поля на поверхности металлического тела при его движении В атмосфрере и ионосфере
}

\section{(C) В.А. Федоров}

Радиотехнический институт им. акад. А.Л. Минца, Москва

E-mail: f_v99@mail.ru

Поступило в Редакцию 29 декабря 2016 г.

Определены величина и знак электростатического заряда, потенциала и напряженности магнитного поля на поверхности металлического тела, движущегося в атмосфере и ионосфере. Показано, что на величину и знак отмеченных выше функций основное влияние оказывают скорость и высота движения тела.

DOI: 10.21883 /PJTF.2017.22.45266.16656

Изучение проблемы электростатического заряжения электрически изолированных металлических тел, движущихся в атмосферном воздухе или плазме, было начато достаточно давно [1,2]. Данное обстоятельство связано с тем, что явление электризации металлических тел широко распространено в природе и технике, причем его возникновение часто приводит к негативным последствиям (см. ссылки в $[1,2])$. В частности, электризация металлических тел в воздушных потоках сильно влияет на процессы при полетах самолетов через облака, осадки и т.п. Например, величина потенциала на поверхности $\varphi$ достигает значений $\varphi \approx 10^{5}-10^{8} \mathrm{~V}$ относительно окружающей среды [3]. Поскольку в работе рассматривается изолированное металлическое тело, его потенциал может быть определен только относительно потенциала окружающей среды, который обычно принимается за нулевой [3].

Приведенные величины $\varphi$, полученные при теоретических и экспериментальных исследованиях электризации металлических тел в воздухе [1-3], оказывают значительное влияние на протекание физических процессов как на поверхности тела, так и в его окрестности. Степень 
влияния в первую очередь зависит от величины электростатического заряда $Q$ на поверхности. Поэтому при достижении телом, движущимся в атмосфере, определенных величин $Q$ становится возможным возникновение таких физических явлений, как коронирование, электрический пробой среды, окружающей тело, ионизация молекул воздуха и т.д. [1-4]. Отмеченные явления возникают в окрестности тела при достижении его потенциалом определенной величины, что приводит к увеличению скорости движения заряженных частиц в его окрестности.

В настоящее время основным механизмом электростатического заряжения металлических тел при их движении в воздухе считается контактная разность потенциалов [1]. Исходя из этого механизма в работе [2] было получено, что при движении в атмосфере металлической сферы радиусом $R=100 \mathrm{~cm}$ на высоте $h=30 \mathrm{~km}$ со скоростью $v=0.2 \mathrm{~km} / \mathrm{s}$ заряд достигает в равновесном $(t \rightarrow \infty)$ состоянии величины $Q=3.3 \cdot 10^{7} \mathrm{CGSE}$, а потенциал $\varphi=Q / R=9.9 \cdot 10^{7} \mathrm{~V}$.

Если движение тела происходит на высотах $h>40 \mathrm{~km}$, то в атмосфере присутствуют ионы и электроны. Например, их концентрация на высоте $h=55 \mathrm{~km}$ равна $N_{i}=N_{e}=10 \mathrm{~cm}^{-3}$, а на высоте $h=70 \mathrm{~km}$ $N_{i}=N_{e}=10^{2} \mathrm{~cm}^{-3}$ [5]. Это приводит к тому, что на поверхность тела течет тепловой ток электронов, уменьшающий положительный заряд $Q$. В работе [6] решена задача об обтекании металлической сферы радиусом $R=100 \mathrm{~cm}$ потоком плазмы, движущейся со скоростью $v=0.2 \mathrm{~km} / \mathrm{s}$ на высоте $h=70 \mathrm{~km}$, где нейтральные частицы представляют собой молекулы воздуха. Было получено, что $Q=58 \mathrm{CGSE}$, $\varphi=174 \mathrm{~V}$.

Возникающий заряд $Q$ на поверхности металлической сферы при ее прямолинейном равномерном движении со скоростью $v$ создает магнитное поле напряженностью

$$
H=\frac{Q v}{r^{2}} \sin \alpha,
$$

где $\alpha$ - угол между вектором скорости $v$ и рассматриваемым направлением, $r$ - расстояние до точки наблюдения. Если положить $\alpha=90^{\circ}$, а $r=R=100 \mathrm{~cm}$, то из (1) можно получить значения $H$ на поверхности сферы в направлении, перпендикулярном вектору скорости $v$ для различных величин $Q$ и $v$. Значения и знак функций $Q$, $\varphi$ и $H$ при движении тела в атмосфере со скоростью $v=0.2 \mathrm{~km} / \mathrm{s}$ 
представим в виде $h=30,70 \mathrm{~km}\left(Q=3.3 \cdot 10^{7}, 58 \mathrm{CGSE} ; \varphi=9.9 \cdot 10^{7} \mathrm{~V}\right.$; $\left.H=6.6 \cdot 10^{5}, 1.2 \mathrm{Oe}\right)$.

Движение металлического тела в атмосфере с гиперзвуковой скоростью приводит к возникновению плазменной оболочки вокруг тела благодаря ионизации молекул воздуха [5]. Из результатов работ $[5,7]$ получим значения концентрации электронов плазмы $N_{e}$ на высотах $h=30,60,90 \mathrm{~km}$ при соответствующих скоростях $v=3,5,7 \mathrm{~km} / \mathrm{s}$, которые представлены следующим образом. При скорости $v=3 \mathrm{~km} / \mathrm{s}$, $h=30,60,90 \mathrm{~km} N_{e}=2.0 \cdot 10^{11}, 2.5 \cdot 10^{9}, 7.0 \cdot 10^{6} \mathrm{~cm}^{-3}$. При скорости $v=5 \mathrm{~km} / \mathrm{s}, h=30,60,90 \mathrm{~km}\left(N_{e}=7.7 \cdot 10^{14}, 1.2 \cdot 10^{13}, 7.0 \cdot 10^{10} \mathrm{~cm}^{-3}\right.$. При скорости $v=7 \mathrm{~km} / \mathrm{s}, h=30,60,90 \mathrm{~km} N_{e}=7.4 \cdot 10^{15}, 7.7 \cdot 10^{13}$, $4.0 \cdot 10^{11} \mathrm{~cm}^{-3}$.

Учитывая приведенные данные и исходя из средней кинетической энергии электронов плазмы, примем, что средние значения кинетической температуры электронов плазмы для высот $h=30,60,90 \mathrm{~km}$ в рассматриваемом случае равны $T=1000,2000,3000 \mathrm{~K}$ соответственно. Для рассматриваемого случая оценим величину теплового тока электронов плазмы $I_{e k}$ на поверхность тела в виде металлической сферы с $R=100 \mathrm{~cm}$. Из формулы

$$
I_{e k}=\frac{1}{4} e N_{e} v_{e k} S,
$$

где

$$
v_{e k}=\sqrt{2 k T / m_{e}}
$$

- тепловая скорость электронов плазмы, $S=4 \pi R^{2}$, е, $m_{e}-$ заряд и масса электрона, $k-$ постоянная Больцмана, получим $I_{e k 1,2,3}=5.2 \cdot 10^{13}, 9.3 \cdot 10^{11}, 3.2 \cdot 10^{9}$.

Полученные значения $/ I_{e k 1,2,3} /$ на несколько порядков превышают величину тока зарядки металлического тела, который можно оценить следующим образом. Принимая, что зарядка тела происходит равномерно, найдем $I_{q} \approx Q / t \approx 5.2 \cdot 10^{5} / 10 \approx 5.2 \cdot 10^{4} \mathrm{CGSE} / \mathrm{s}$ (см. рис. 2 в [2]). Таким образом, в данном случае электризацию тела на основе контактной разности потенциалов можно не учитывать. Следовательно, при движении в атмосфере с гиперзвуковой скоростью металлическое тело будет приобретать электростатический заряд отрицательного знака благодаря разности тепловых скоростей электронов и ионов. С учетом

Письма в ЖТФ, 2017, том 43, вып. 22 
Величины и знак функций $Q, \varphi$ и $H$ ИСЗ

\begin{tabular}{c|c|c|c|c}
\hline $\begin{array}{c}h, \\
\mathrm{~km}\end{array}$ & $T, \mathrm{~K}$ & $\begin{array}{c}Q, \\
\text { CGSE }\end{array}$ & $\varphi, \mathrm{V}$ & $H, \mathrm{Oe}$ \\
\hline 250 & 1700 & -0.05 & -0.15 & -4.0 \\
500 & 2600 & -0.07 & -0.23 & -5.7 \\
1000 & 3000 & -0.09 & -0.27 & -7.3
\end{tabular}

отмеченного, найдем, что

$$
\begin{gathered}
\varphi=k T / e, \\
Q=R \varphi .
\end{gathered}
$$

Учитывая формулу (1), где примем $v=3 \mathrm{~km} / \mathrm{s}$, получим значения $H$ в данном случае. Найденные величины и знак функций $Q, \varphi$ и $H$ в зависимости от $h=30,60,90 \mathrm{~km}$ и $v(T=1000,2000,3000 \mathrm{~K})$ при движении в атмосфере с гиперзвуковой скоростью представим следующим образом: $Q=-0.03,-0.06,-0.09 \mathrm{CGSE} ; \varphi=-0.09,-0.18,-0.27 \mathrm{~V} ; H=-9$, $-18,-27$ Oe.

Определим значения и знак функций $Q, \varphi, H$, которые имеют искусственные спутники Земли (ИЗС), движущиеся в ионосферной плазме со скоростью $v \approx 8.1 \mathrm{~km} / \mathrm{s}$ на высотах $h=250,500,1000 \mathrm{~km}$. Поскольку на рассматриваемых высотах в ионосфере концентрация нейтральных частиц на много порядков меньше, чем в атмосфере, механизм электростатического заряжения, в основе которого лежит контактная разность потенциалов, на данных высотах невозможен. Поэтому на отмеченных высотах действует механизм заряжения металлического тела, основанный на разности величин тепловых скоростей электронов и ионов плазмы или разности величин кинетических температур заряженных частиц плазмы [8].

На данных высотах в дневное время кинетическая температура электронов плазмы равна $T=1700 \mathrm{~K}(250 \mathrm{~km}), 2600 \mathrm{~K}(500 \mathrm{~km})$, $3000 \mathrm{~K}(1000 \mathrm{~km})[8]$. Принимая, что ИСЗ имеет форму сферы из металла радиусом $R=100 \mathrm{~cm}$, и, используя принятые значения $T$, определим величины функций $Q, \varphi, H$ и их знак с помощью формул (1), (4), (5). Найденные величины представим в таблице.

Знак функций $Q, \varphi, H$ в таблице показывает, что они имеют отрицательные значения и достаточно малы по сравнению со значениями 
аналогичных функций в случае движения металлического тела со скоростью, меньшей звуковой в атмосфере. Однако необходимо отметить, что на ИСЗ при попадании его в тень Земли [9] или в поток заряженных частиц от солнечных вспышек [9] возникают электростатические заряды (в основном отрицательные), создающие электрические потенциалы величиной $\varphi \approx(1-10) \mathrm{kV}$ [9], при которых возможно возникновение электростатических разрядов или пробой среды.

Необходимо отметить, что при рассмотрении физических процессов, имеющих место в настоящей работе, использовались средние значения параметров частиц атмосферы и ионосферы (см. приведенную литературу).

\section{Список литературы}

[1] Имянитов И.М. // ДАН СССР. 1958. Т. 121. № 1. С. 93-96.

[2] Имянитов И.М., Старовойтов А.Т. // ЖТФ. 1962. Т. 32. В. 6. С. 759.

[3] Имянитов И.М. Электризация самолетов в облаках и осадках. Л.: Гидрометеоиздат, $1970.211 \mathrm{c}$.

[4] Добрецов Л.Н., Гомоюнова М.В. Эмиссионная электроника. М.: Атомиздат, 1966. $564 \mathrm{c}$.

[5] Мартин Джс. Вход в атмосферу. М.: Мир, 1969. 320 с.

[6] Федоров В.А. // Физика плазмы. 2014. Т. 40. № 10. С. 946-952.

[7] Федоров В.А. // ЖТФ. 2016. Т. 86. В. 5. С. 148-150.

[8] Альперт Я.Л., Гуревич А.В., Питаевский Л.П. Искусственные спутники в разреженной плазме. М.: Наука, 1964. $384 \mathrm{c}$.

[9] Искусственные пучки частиц в космической плазме / Под ред. Б. Гранналя. М.: Мир, 1985. $456 \mathrm{c.}$

Письма в ЖТФ, 2017, том 43, вып. 22 